\title{
Téoros
}

Revue de recherche en tourisme

\section{Écotourisme et conservation de la biodiversité dans les parcs nationaux du Québec}

Le parc marin du Saguenay-Saint-Laurent

\section{Maryse Grandbois}

Volume 18, numéro 2, été 1999

La nordicité

URI : https://id.erudit.org/iderudit/1072082ar

DOI : https://doi.org/10.7202/1072082ar

Aller au sommaire du numéro

Éditeur(s)

Université du Québec à Montréal

ISSN

0712-8657 (imprimé)

1923-2705 (numérique)

Découvrir la revue

Citer cet article

Grandbois, M. (1999). Écotourisme et conservation de la biodiversité dans les parcs nationaux du Québec : le parc marin du Saguenay-Saint-Laurent. Téoros, 18(2), 24-29. https://doi.org/10.7202/1072082ar d'utilisation que vous pouvez consulter en ligne. 


\title{
ÉCOTOURISME ET CONSERVATION DE LA BIODIVERSITÉ DANS LES PARCS nationauX du Québec
}

\author{
LE PARC MARIN DU SAGUENAY-SAINT-LAURENT
}

\section{Maryse Grandbois}

Depuis l'aménagement du premier parc national canadien, celui de Banffen 1887 , le réseau fédéral des parcs nationaux a largement contribué à soutenir l'essor et le développement de l'industrie touristique. Les objectifs de conservation de la nature et de la biodiversité, apparus beaucoup plus tard, remontent à l'adoption, en 1964, d'une première politique consenvationniste pour le réseau des parcs. À partir de ce point tournant, les politiques federales de gestion des parcs poursuivent deux objectifs : sauvegarder le patrimoine naturel et permettre sa mise en valeur à des fins touristiques.

Aujourd'hui, le ministère du Patrimoine canadien, responsable du réseau des parcs, a non seulement pour mission de a sauvegarder a jamais des aires naturelles représentatives d'intếrêt canadien $\%$, mais également de * favoriser chez le public la connaissance, l'appréciation et la jouissance de ce patrimoine naturel $\%$ (Ministère du Patrimoine canadien, $1997: 1$ ). Les politiques et les lois sur les parcs et les aires marines de conservation traduisent ces objectifs : elles visent à conserver l'intégrité écologique des parcs tout en permettant au public de les découvrir et de les utiliser. Pour réaliser ces objectifs, le gouvernement, le ministere et le gestionnaire des parcs, Parcs Canada, misent sur la participation du public, une participation devenue la a pierre angulaire de la politique, de la planification et des pratiques de gestion $w$ (Ministère du Patrimoine canadien, $1994: 4$ ).

Au cours des 35 dernières années, on a toutefois difficilement concilié les missions touristique et conservationniste ; en outre les effets du tourisme menacent toujours diverses especes et leur habitat dans plusieurs pares nationaux. Dans ce contexte, l'écotourisme fait figure de voie de solution. Défini rigoureusement, l'ecotourisme stimule l'amenagement des parcs et la conservation des ressources, tout en apportant des bénéfices aux communautés locales (Boo, $1991: 4$; Agardy, 1993 : 219). En principe, les aménagements demeurent légers : on planifie des infrastructures minimales d"acces et de service à l'intérieur des limites des parcs, alors qu'on offre davantage d'activités et de services dans la zone périphérique. La mise en ouvre de programmes d'éducation et de sensibilisation de la population ainsi que contribuent à atténuer les effets sur l'environnement des diverses activités, mais les pressions sur les ressources demeurent considérables.

Nous cherchons à savoir si l'écotourisme et la conservation de la diversité biologique dans les parcs nationaux et les aires marines de conservation peuvent coexister avec succès. Nous présenterons d'abord les politiques publiques et le droit relatifs à la création et la gestion des parcs nationaux pour traiter ensuite d'un cas particulier, celui du parc marin du SaguenaySaint-Laurent. Nous tenterons de dégager quelques éléments caractéristiques du droit l'adoption de certaines mesures restrictives canadien et des solutions apportées par les gestionnaires des parcs nationaux en vue de réaliser leurs objectifs.

\section{LES POLITIQUES PUBLIQUES RELATIVES AUX PARCS NATIONAUX}

Le réseau des parcs nationaux est d'abord fondés sur des critères de représentativité du patrimoine canadien : il doit représenter un échantillonnage des 39 régions naturelles canadiennes identifiées par le ministère. A ce jour, $60 \%$ de ce réseau est complété, couvrant $2 \%$ de la superficie du territoire canadien, avec 38 parcs aménagés et 5 en voie de création. Quatre de ces pares sont situés sur le territoire du Québec : les parcs de la Mauricie et Forillon (1970), la réserve du parc national Archipel-de-Mingan (1993) et le parc marin du Saguenay-Saint-Laurent (1998).

L'administration du réseau est confiée à Parcs Canada, organisme public relevant depuis deux ans d'un nouveau ministère, le ministère du Patrimoine canadien. S'appuyant à la fois sur la Convention internationale sur la diversité biologique et la Convention de l'UNESCO sur la conservation du patrimoine mondial naturel et culturel, Parcs Canada doit gérer les ressources naturelles sous sa responsabilité à la manière de biens culturels. Ainsi, doit-il:

Créer un réseau complet d'aires patrimoniales protégées représentatives du patrimoine naturel et culturel du Canadal... / gérées selon les principes et pratiques de gestion des ressources culturelles, de sorte que leur intégrité commémorative sera 


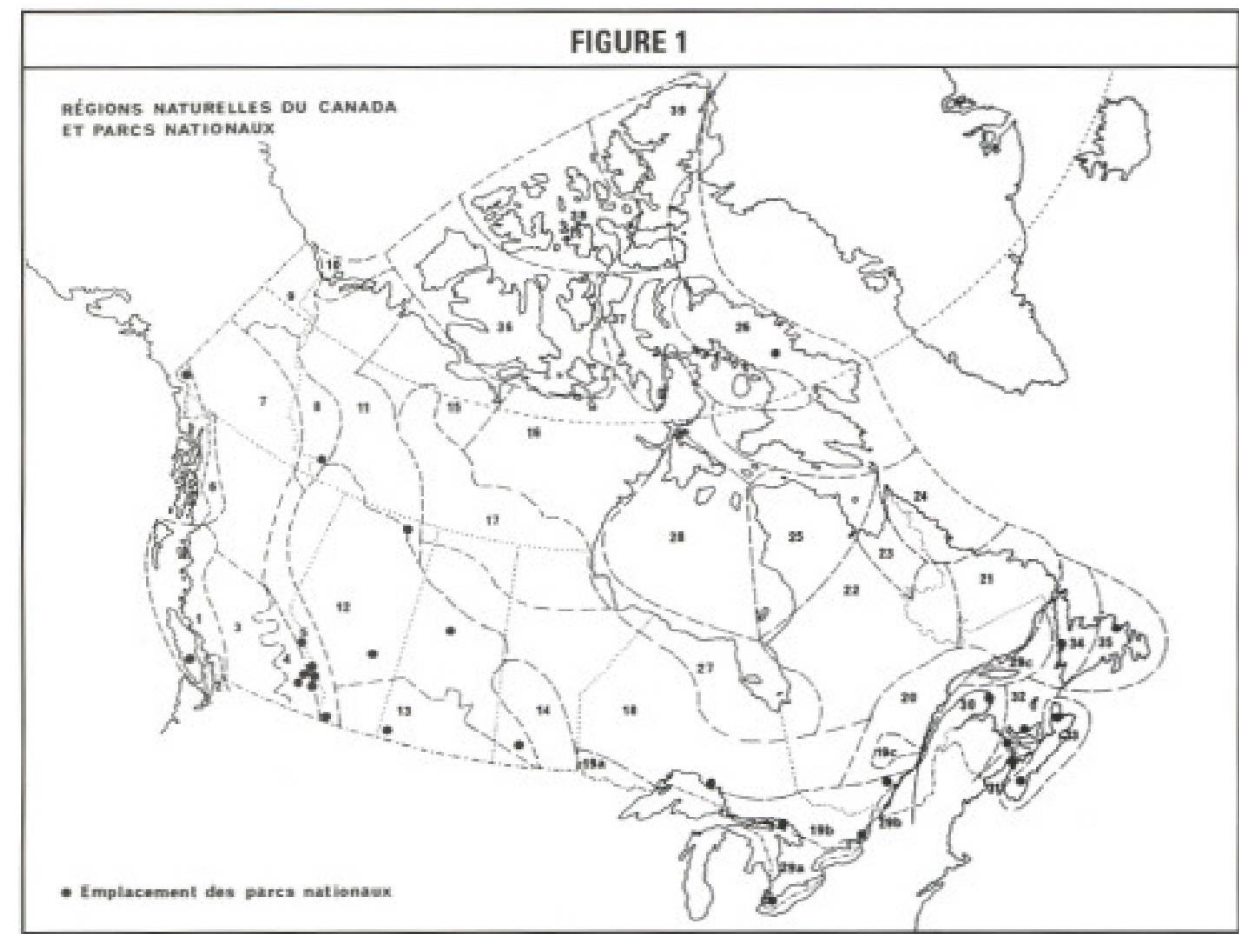

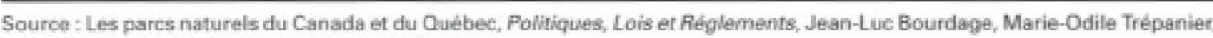
Université de Montréall, Institut Botaniquue, 1984

assurée et que la vie socio-économique des communautés où elles sont situées sera amelioré (Ministère du Patrimoine canadien, 1994 : 10).

Cette conception de l'aire protégée représentative du patrimoine et investie d'une mission culturelle, voire idéologique ${ }^{1}$, marque tout le réseau, qu'il s'agisse de la création de parcs terrestres ou d'aires marines. Dans les parcs, l'écologie, la culture et le tourisme se rejoignent pour assurer la représentation et la mise en valeur d'un patrimoine collectif canadien.

\section{L'AMÉNAGEMENT DES PARCS NATIONAUX}

La création d'un parc national exige d'abord la coopération des autorité́s provinciales ou territoriales et des autochtones concernes, ainsi que la participation des différents groupes d'intérêt régionaux. La première étape, lä sélection d'un site afin de représenter l"une des 29 aires naturelles déterminées par le ministère, est suivie d'une étude de faisabilité réalisée avec la participation des gouvernements et des populations du territoire. Vient ensuite une entente entre les gouvernements quant a la création formelle du parc ; cette entente sera entérinée par voie législative, par une modification à la Loi sur les parcs nationawx.

L'entente administrative doit prévoir l'ensemble des conditions d'établissement et d'aménagement du parc. Elle porte non seulement sur les limites définitives du parc, le partage des coûts d'acquisition des terres entre les paliers de gouvernement et les termes du transfert des terres, mais aussi sur la poursuite d'activités traditionnelles d'exploitation des ressources renouvelables par les autochtones, le cas échéant, de même que sur la planification et la gestion du parc et de sa périphérie, la composition et le rôle du conseil de gestion du parc, l'intégration régionale et les avantages économiques locaux. Notons que le plan de gestion du parc doit être entrepris dès que les terres sont réservées : qu'il doit être approuvé par le ministre et déposé devant le Parlement et par la suite qu'il doit être révisé tous les cinq ans.

Dans l'ensemble du réseau des parcs, la protection des ressources culturelles et ćcologiques et l'accès du public aux services sont assurés par le recours à des techniques de zonage. De manière générale, à moins qu'il ne soit nécessaire de procéder autrement en raison de la fragilité des écosystemes, cinq types de zones sont aménagées :
1. Préservation speciale, pour assurer la protection d'habitats ou d'especes menacées ;

2. Milieu sauvage, où sont aménagées des réserves intégrales;

3. Milieu naturel, où sont autorisées certaines activités à faible incidence sur l'environnement, telles les balades en montagne ;

4. Loisirs de plein air, où sont aménagées des aires de loisirs, selon la situation et les objectifs du parc :

5. Services du parc, où sont regroupées les principales infrastructures. Dans les parcs récemment crées, les bâtiments administratifs sont localisés à l'extérieur des limites du pare et des services plus diversifiés sont offerts dans les collectivités locales adjacentes.

La délimitation de ces zones répond à deux objectifs : conserver les ressources et sauvegarder a la qualité de l'expérience des visiteurs $m$ (Ministère du Patrimoine canadien, $1994: 38-39$ ). Axés sur a la compréhension, l'appréciation et la jouissance du patrimoine naturel et culturel , les aménagements des parcs sont conçus pour satisfaire aux attentes du public, tout en respectant des principes directeurs de qualité de l'environnement. Ces principes exigent le maintien de $\alpha$ l'intégrité écologique et commémorative $\%$, par la gestion des ressources et l'arbitrage des activités conflictuelles, la mise en ceuvre de programmes d'éducation, d'interprétation et de communication, la recherche scientifique, ainsi que la conception d'activités appropriées à l'intention des visiteurs. $S^{\prime}$ appuyant sur la coopération des visiteurs, des organismes et des groupes régionaux, les gestionnaires doivent rendre compte au Ministère et au Parlement $\alpha$ de l'application et du respect de ces principes et de la mise en ouvre des politiques relatives aux activités ". La Commission sur l'intégrité écologique des parcs nationaux au Canada, formée en 1999 à l'occasion du $10^{\circ}$ anniversaire de l'adoption de ces principes, prépare un bilan général de la gestion des écosystèmes des parcs.

Rappelons que traditionnellement, en Amérique du Nord, la création d'un parc national exigeait la réunion de certaines conditions, au nombre desquelles figure l'absence d'habitation humaine et d'exploitation des ressources. Le réseau des parcs nationaux s'est peu à peu écarté de 
ce modèle classique, composé essentiellement d'espaces inhabites ou d'aires isolées, difficiles d'accès ou de faible intérêt économique. Ainsi, l'aménagement du pare national Forillon, sur le littoral gaspésien, a entraîné l'expropriation de 200 familles et le parc Kouchibougouac, au Nouveau-Brunswick, a été délimité malgré l'opposition d'une partic de la population locale. Établis sur des zones côtières, ces deux parcs visaient notam= ment le développement du tourisme.

Même si, à la fin des années 1980, on comptait quelques parcs nationaux établis sur des zones côtières au Canada, tels Forillon, Pacific Rim, Kouchibougouac et Auyuittuq, il faudra attendre jusqu'en 1987 pour que le gouvernement fédéral se dote d'une politique des pares marins afin de * protéger et conserver des aires marines représentatives du littoral canadien, a l'intention des générations actuelles et futures $\%$ (Environnement Canada, $1987: 5$ ). L'aménagement intégré, la conservation de la diversité biologique et l'écotourisme vont devenir les maitres-mots de ces nouvelles aires protégées.

\section{LES AIRES MARINES DE CONSERVATION}

Premier état cốtier au monde avec 243000 km de périmètre côtier, le Canada n"a pas manifesté beaucoup d'empressement aे constituer son réseau de parcs marins et ce, malgré la fragilité des zones côtières de certaines régions. C'est en 1988 que la Loi sur les parcs nationaux a été modifiée pour faire place au concept et à la définition de pare marin. La loi modificatrice élargissait la définition des terres publiques au domaine maritime et autorisait l'Administration a octroyer des baux et des permis d'occupation des terres riveraines. Les nouvelles dispositions exigeaient également le dépôt de plans de gestion et prévoyaient la conclusion d'ententés fédérales-provinciales pour la création et l"administration des parcs marins. La Loi sur la marine marchande, la Loi sur la prévention de la pollution des eaux arctiques et la Loi sur le pilotage ont par la suite été modifiées à leur tour; aux fins de contrôler la circulation maritime aux abords des pares marins.

Dans le but de constituer son réseau, le gouvernement fédéral a opté, comme pour les parcs nationaux, pour la méthode de l'échantillonnage régional. Ainsi, le ministère de l'Environnement a-t-il inventorié 29 régions naturelles marines au Canada, où il projette d'aménager un parc marin avant l'an 2010 (Ministère du Patrimoine canadien, 1995). La procédure de création de tels parcs ne differe pas de celle des parcs terrestres: la sélection des sites représentatifs est suivie d'une étude de faisabilité, puis d'une entente fédéraleprovinciale et, enfin, d'une loi constitutive du parc marin. De manière générale, les pares sont proprićtés du gouvernement fédéral, mais les compétences législatives se partagent entre les deux paliers de gouvernement de sorte que des ententes bilatérales gèrent le contrôle des pollutions, l'aménagement des ressources et la maîtrise des flux touristiques.

La politique canadienne des parcs marins de 1987, antérieure à la loi, visait avant tout à favoriser la connaissance et la jouissance des pares marins par le public. Ainsi, la peche, la navigation, la gestion des ressources et le transport maritime s'y poursuivaient normalement, sous réserve de la réglementation de zonage. En 1994, à la suite des directives de l'UICN (l'Union mondiale pour la nature) sur les parcs marins (1992), le gouvernement fédéral a restructure et clarifié sa politique des parcs marins, devenus des a aires marines de conservation $\%$. La gestion de ces aires marines poursuit depuis un objectif d'utilisation durable des ressources, de sorte que toute exploitation des ressources du sous-sol est désormais interdite.

Selon les termes de la nouvelle politique, la planification et la gestion des parcs marins doivent différer des pratiques des parcs terrestres pour prendre en compte * la nature particulière des écosystèmes marins, ouverts, vastes et dynamiques x, englobant à la fois le fond marin, le soussol et la colonne d'eau (Ministère du Patrimoine canadien, 1994 : 48-50). De plus, compte tenu de la complexité des lois et des sphères d'attribution de compétence en milieu marin - la politique répertorie 36 lois fédérales et 20 lois provinciales et territoriales - le ministère pilote l'adoption d'une nouvelle loi et de nouveaux règlements sur les aires marines de conservation (Ministère du Patrimoine canadien, $1994: 49$ ).

Les politiques de création des parcs tentent également d'associer les populations locales, leur participation ayant ếté iden- tifiée comme principal facteur de succès des programmes de protection, tel qu'en témoignent des expériences récentes de création de pares marins ${ }^{2}$. $\mathrm{A}$ la suite de ces constats, un projet canadien de parc marin a été abandonné en raison de l'opposition des pêcheurs et des aquaculteurs de la baie de Fundy, puisque ceux-ci craignaient à la fois les expropriations massives et les effets du tourisme sur la peche côtière (Butler, 1994 : 125-134). Le projet de parc marin de Bona Vista, à TerreNeuve, a dû être retiré en 1999 pour les mêmes raisons, si bien que les études de faisabilité de Parcs Canada, traditionnellement orientées vers l'écologie des parcs, s'apprêtent à adopter une méthodologie différente pour prendre en compte les aspects socio-économiques dès la phase de conceptualisation d'un pare marin.

En 1999. le Canada ne compte cependant que trois parcs marins, tous conçus avant l'adoption de la politique de 1994: Fathom Five (Ontario, 1987), Gwai Haanas (Colombie-Britannique, 1988) et SaguenaySaint-Laurent (Québec, 1990). Ces parcs ont contribué à développer le tourisme de croisière au Canada (OCDE, 1989 : 135136 ; Miller, 1993: 181-189) tout en exigeant des aménagements importants, à l'extérieur des limites du parc comme à l'intérieur. En ce sens, ils perturbent considérablement les zones côtières, traditionnellement très peu aménagées en dehors des zones portuaires. Pour minimiser les effets sur l'environnement, les biologis= tes proposent la mise en cuvre de programmes d'éducation et de sensibilisation ainsi que l'adoption de certaines mesures de contrôle : limite du nombre de visiteurs, dispersion des visiteurs dans les parcs. restriction d'accès à certaines périodes de l'année. Ils incitent également les états à réserver des superficies de plus en plus grandes pour éviter de fragmenter les aires protégées (Alexander, $1993: 186$ ), ce qui a pour effet d'exiger la mise en ceuvre de nouvelles formes de planification spatiale intégrée et la gestion d'activités souvent conflictuelles.

\section{LE PARC MARIN DU SAGUENAY-SAINT-LAURENT}

L'environnement côtier illustre à̀ merveille combien le contrôle des pollutions et la conservation de la diversité biologique sont totalement interdépendants. En ce sens, la zone côtière est un fait total *, au sens 
où les sociologues l'entendent. Le littoral fait également l'objet de multiples interventions, à cause de la diversité des sources de pollution et de leur effet de synergiè, à cause aussi de l'ensemble des activités qui s'y pratiquent. Dans cette perspective, l'écotourisme, bien planifié et bien géré, peut contribuer à assurer la protection de l'environnement, tout en générant des retombées économiques régionales, compte tenu de l'attraction touristique qu'exercent les zones côtières. Rappelons que certains éléments facilitent la gestion des pares et favorisent la conciliation des objectifs de conservation et de récréation. Les facteurs de succès tiennent le plus souvent à l'étendue des pouvoirs administratifs des gestionnaires et a l'ampleur de la participation publique, de même qu"à la cohérence spatiale et à la coordination institutionnelle. Ces divers facteurs doivent toutefois être soutenus par un régime de responsabilité pénale et civile ainsi que par un recours conjugué aux stimulants économiques et à la réglementation (Leitman, 1998: 129-143). Dans le cas des pares marins, I'aménagement intégré des zones côtières tend à s'imposer comme étant la voie de solution, ces pares fournissant un cadre concret à l'aménagement intégré des ressources et des activités qui se pratiquent sur le littoral. On mise alors sur la participation active des différents acteurs sociaux : autorités gouvernementales, personnel du parc, communautés locales, industrie touristique, $\mathrm{ONG}$ locales, institutions financières, consommateurs et usagers.

Le parc marin du Saguenay-Saint-Laurent, dont la création a été planifiée au début des années $1990, \mathrm{~s}^{\prime}$ inscrit dans cette perspective de conciliation des objectifs de l'écotourisme et de la conservation de la diversité biologique. Nous nous intéresserons ici aux moyens juridiques utilisés pour mettre en place et réaliser ce double objectif, à la fois dans les textes relatifs à l'aménagement de ce parc marin et dans la gestion des activités touristiques qui s'y pratiquent.

\section{L'AMÉNAGEMENT DU PARC MARIN}

Le pare marin du Saguenay-Saint-Laurent a fait l'objet d'une entente fédérale-provinciale en date du 6 avril 1990. Selon les termes de cette entente, des représentants des gouvernements fédéral et québécois président à la gestion du parc ; les deux paliers de gouvernement définissent conjointement les politiques de concertation, de suivi, de mise en valeur et de conservation. Ils s'entendent également pour favoriser l'intégration de ce parc marin au milieu régional et pour développer la recherche scientifique. Un comité consultatif, constitué de représentants des ministères, de l'UICN, de la Coalition pour le pare marin et de scientifiques, contribue au développement des objectifs et à la planification du parc, de même qu'à l'harmonisation des lois et des règlements et à la conception des plans d'urgence.

À la suite de l'entente, le ministère du Patrimoine canadien et le ministère du Loisir, de la chasse et de la pêche du Québec ont tenu des audiences publiques sur les limites du parc marin. Une nouvelle consultation publique a eu lieu en 1993 sur la proposition de mise en valeur du parc. Le plan directeur est issu de l'ensemble des consultations. À la fin du processus, deux lois, l'une fédérale ${ }^{3}$ et l'autre provinciale ${ }^{4}$, entrées en vigueur en juin $1998^{5}$, ont concrétisé la création et l'aménagement du pare marin. Chacune des lois met en place un régime administratif et un régime pénal de sanction des infractions.

Constitué d'une partie représentative du fjord du Saguenay et de l'estuaire du SaintLaurent ${ }^{6}$, d'une superficie totale de $1138 \mathrm{~km}^{2}$, le parc vise à instituer une gestion intégrée de la zone côttière du fleuve Saint-Laurent, aux confluents de la rivière Saguenay. Pour ce faire, l'aménagement du parc poursuit des objectifs généraux et particuliers liés non seulement à la conservation des ressources, à l'éducation et à l'interprétation et la recherche scientifique, mais aussi à l'utilisation et à la mise en valeur du territoire et à son intégration harmonieuse au milieu régional. La loi fédérale, par exemple, a pour objectif de * rehausser, au profit des générations actuelles et futures, le niveau de protection des écosystèmes d'une partie représentative du fjord du Saguenay et de l'estuaire du Saint-Laurent aux fins de conservation, tout en favorisant son utilisation à des fins éducatives, récréatives et scientifiques $s^{7}$.

Un comité de gestion assiste la direction du parc pour assurer la concertation et la complémentarité des activités, l'harmonisation des normes et le suivi du plan directeur. Ce plan directeur doit être réexaminé tous les sept ans et les limites du parc peuvent être révisées, avec l'accord des gouvernements fédéral et québécois. Quatre types de zones sont délimitées dans le pare:

- La Zone I, de préservation intégrale, où seules les activités scientifiques peuvent être autorisées.

- La Zone II, de protection spécifique, où sont autorisées les activités récréatives sans prélèvement et les activités éducatives.

- La Zone III, de protection générale, où sont autorisées toutes les activités récréatives et éducatives définies pour le parc.

- La Zone IV, d'utilisation générale, où sont également pratiquées la circulation maritime, la pêche commerciale et la chasse à la sauvagine.

Le parc marin du Saguenay-Saint-Laurent comprend trois aires : le fjord, l'estuaire et une zone de confluence. Dans chacune de ces aires, on distingue des zones de préservation intégrale et de protection spécifique, des zones d'ambiance et, enfin, des zones d'utilisations spécifiques, dans lesquelles sont organisées des croisières, des activités de récolte des coquillages, où la pêche sportive, la pêche commerciale et l'aquaculture sont autorisées, de mềme que la chasse à la sauvagine et la navigation.

L'aire de coordination du parc marin comprend les municipalités riveraines et plusieurs autres collectivités locales, situées en amont du fjord et sur la rive sud de l'estuaire, de Chicoutimi à Tadoussac. Comme le souligne le plan directeur, cette région, * très développée sur le plan touristique *, est à la fois « habitée depuis longtemps et fortement utilisée à des fins industrielles et commerciales $\%$ (Ministère du Patrimoine canadien, ministère de l'Environnement et de la faune du Québec, 1995 : 47). L'aménagement du parc doit donc prendre en compte l'ensemble des activités économiques, des particularités du site et des intérêts des habitants et des visiteurs, tout en tentant de favoriser l'écotourisme et d'assurer la protection de la biodiversité.

\section{LA CONSERVATION DE LA BIODIVERSITÉ}

Rappelons d'entrée de jeu que l'environnement du parc marin du Saguenay-Saint- 
Laurent, composế de systèmes aquatiques très ouverts aux flux d'énergie extếrieurs, est encore mal connu. De manière générale, la chimic, la physique et la biologic de ces milieux n'ont fait l'objet que de recherches ponctuelles et fragmentaires, du moins avant le début des années $1990^{\circ}$. La région du parc marin compte 6 écosystèmes distincts, soit 12 habitats, porteurs de nombreuses espèces (Centre SaintLaurent, 1991 : 1). La concentration inhabituelle de 4 espèces de baleine - le béluga, le petit rorqual, le rorqual bleu et le rorqual commun - attire jusqu'à 250000 visiteurs par an (Vézina, $1997: 26$ ). Les milieux marin et aquatique sont contaminés par de multiples rejets industriels et, en 1988, la région enregistrait plus de 19000 mouvements de navires (Centre Saint-Laurent, $1991:$ 4).

Le béluga a été classé espèce menacée en 1983 par le Comité sur les espèces fauniques menacées d'extinction au Canada. Aujourd'hui, malgré la mise en cuvre de programmes de protection, la situation du béluga demeure précaire, en raison de la contamination du milieu et des dérangements physiques et sonores qu'il subit, de la navigation de plaisance au tourisme commercial en mer, de l'aménagement des voies d'eau aux activités de recherche scientifique. Notons que les baleines attirent jusqu'à 37 bateaux en mểme temps, de $9 \mathrm{~h}$ à 16 h, durant la saison touristique et que l'effet cumulatif de ces dérangements est inconnu ${ }^{9}$. Une population si faible - environ 500 bélugas - se montre particulièrement sensible aux perturbations de son environnement et on estime qu'elle peut développer des problèmes génétiques (Breton et al, 1990:2 ; Hamel, 1996: 117).

Le zonage du parc marin ne suffit pas à protéger le béluga et ses habitats contré les dérangements et encore moins contre la contamination du milieu marin. Compte tenu du caractère ouvert des systèmes aquatiques : « seull un objectif de zéro rejet de substances toxiques pourrait assurer la survie du béluga (Hamel, 1996 : 122). De plus, bien que divers modes de contrôle des dérangements aient été mis en ouvre, tels les restrictions temporelles et les itinéraires obligatoires, ceux-ci se sont montrés jusqu'à maintenant sans effet sur la population de bélugas et l'état de santé des spécimens.

L'aménagement du parc marin a toutefois permis de développer de nouveaux sites

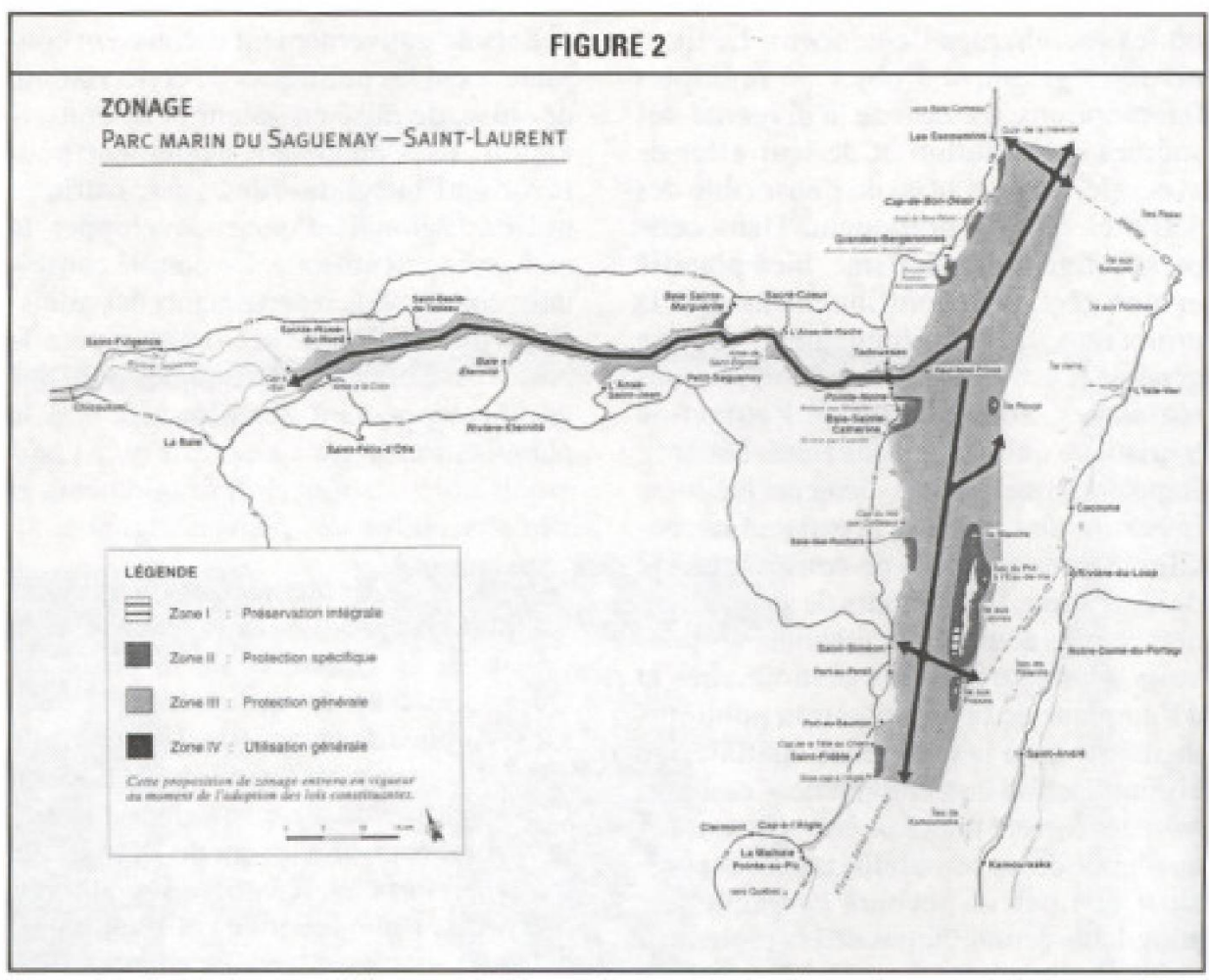

Source : Carrefour de vie, source d'echanges et de richesses, le resume du plan directeur, le parc marin du Saguenay-Saint Lauren, ministre du Patrimoine, Ottawa, 1995

d'observation des baleines - sans dérangement ceux-là - à partir des promontoires de Pointe-Noire et de Cap-de-Bon-désir (Maltais, $1995: 54$ ) et de canaliser les flux touristiques. Cet aménagement s'est fait autour d'une thématique globale : Le parc marin du Saguenay-Saint-Laurent : carrefour de vie, source d'échanges el de richesse. En adoptant ce concept de mise en valeur, les gestionnaires se sont donné pour mots d'ordre : le respect des acquis régionaux, la diversification des activités selon le potentiel des sites, le contrôle des conséquences de la fréquentation des visiteurs sur le milieu marin et la prise en charge par des tiers de certains développements touristiques (Ministère du Patrimoine canadien, ministère de l'Environnement et de la faune du Québec, $1995: 47$ ).

La thématique carrefour de vie, source d'echanges et de richesse se développe autour de 3 pôles phares et de 5 pôles thematiques, relies par un circuit terrestre et maritime. Établis sur les sites de BaieSainte-Catherine, de Cap-de-Bon-Desir et Baie-Éternité, les pôles phares représentent les trois secteurs du parc, le fjord du Saguenay, l'estuaire du Saint-Laurent et la zone de confluence, alors que les pôles thématiques, aménagés en périphérie de ces sites, permettent d'illustrer des thèmes à la fois précis et complémentaires, tels les habitats critiques du béluga, la relation entre l'être humain et la mer ou entre la ville et la mer. Le parc compte également 13 pôles de découverte, où sont organisées des activités récréatives de contact avec le milieu marin, ainsi que 6 carrefours d'accueil et d'orientation, localisés à chacune des entrées du parc. Pour animer les pổles thématiques et les pôles de découverte, les gestionnaires ont conclu des ententes avec des groupes et des communautés locales, tels le Centre écologique de Port-au-Saumon, le Centre d'interprétation de Grandes-Bergeronnes et les collectivités locales riveraines, tout en s'appuyant sur le réseau touristique déjà existant.

Le parc marin du Saguenay-Saint-Laurent s'étend sur une partie représentative du fjord et de l'estuaire, mais sa superficie ne permet pas de protéger des écosystèmes: à titre d'exemple, le parc marin de la Grande Barrière de Corail en Australie, qui sert souvent de modèle au droit comparé, couvre une superficie de $35000 \mathrm{~km}^{2}$ et les pares américains des Keys et de Monterey comptent respectivement 9580 et 13800 $\mathrm{km}^{2}$ (Agardy, $1993: 234-235$; Ministère du Patrimoine canadien, $1995 ; 8$ ). A cet égard, compte tenu de sa faible superficie de $1138 \mathrm{~km}^{2}$, le parc du Saguenay-SaintLaurent doit recourir simultanément à 
l'évocation et à la démonstration pour sensibiliser la population au monde marin (Maltais, 1995: 54). Misant à la fois sur un environnement sain et un espace bien aménagé et géré, l'écotourisme peut alors contribuer à la conservation des aires protégées.

Le plan directeur du parc marin du Saguenay-Saint-Laurent répond à certains critères de suceds des programmes d'écotourisme. Ainsi, le plan de zonage tend à diriger l'expérience touristique vers des éléments caractéristiques de l'environnement et à limiter l'effet du tourisme grâce à des aménagements légers ; de plus, les gestionnaires du parc recourent à des programmes d'éducation à l'environnement pour sensibiliser les visiteurs et les communautés locales à 1 'importance de la conservation (Leitmann, $1998: 129-143$ ). Cependant, pour assurer la conciliation des objectifs, il reste encore à intégrer davantage le parc marin au développement régional et à favoriser une plus grande participation de la population locale.

Les parcs marins ne peuvent poursuivre un objectif de conservation de l'intégrité écologique à la manière des parcs terrestres, à cause de l'importance des activités humaines et économiques qui s'y pratiquent. La gestion intégrée des zones côtières apparaît alors comme solution privilégiée, à la fois pour arbitrer l'ensemble des conséquences et concilier les objectifs touristiques et conservationnistes.

Plusieurs élements de support doivent cependant être réunis pour soutenir les nouveaux modes de contrôle et d'arbitrage. Parmi ces éléments, il faut souligner l'importance de la participation des associa= tions touristiques publiques et privées, la nécessité d'un encadrement législatif et réglementaire approprié, assorti d'un programme de suivi des objectifs et de promotion des activités écotouristiques (Inskeep, 1991: 418-420). Rappelons toutefois que très peu de modèles de gestion intégrée des parcs marins sont actuellement proposés. De plus, des standards ont été développés pour l'écotourisme. mais dans les aires terrestres protégées seulement.

L'approche canadienne prend dès lors tout son intérêt. La définition juridique des parcs marins et l'engagement des communautés côtières dans la création et la gestion des parcs different d'un pays à l'autre, de même que les solutions apportées à la transposition de techniques juridiques $\alpha$ terrestres $\%$, tel le zonage. A cet égard, au Canada, le zonage demeure la principale technique d'aménagement du territoire et de gestion des activités conflictuelles, dans les parcs marins comme dans les parcs terrestres.

Maryse Grandbois est professeure de droit de l'environnement à I'Institut des Sciences de l'environnement de I'UOAM. Elle participe à whe recherche partage Guadeloupe-HaintQuébec sur l'écotourisme et les aires protégées.

\section{NOTES}

1 Le réseau des parces nationaux met en évidence l'interdépendance entré les êtres humains et l'environnement, \& [... ]les valeurs patrimoniales du Canada devraient donc être de mieux en mieux reconnues comme faisant partie d'une éthique qui rassemble tous les Canadiens * (Ministère du Patrimoine canadien, $1994: 10$ ).

2 Pour avoir associé les populations locales des le début du projet, on considere que le pare marin de la baie de Fagatele (Samoa) est un suceès, alors que le pare marin de La Paguera (Porto Rico) n'a pu être aménagé en raison de l'opposition de la population locale (Fiske, $1992: 25$ ).

3 Loi sur le parc marin du Saguenay-Saint-Laurent, L.C. 1997, c. 37.

4 Loi sur le pare marin du Saguenay-Saint-Laurent, L.Q. 1997, c. 16.

5 Décret fixant au 8 juin 1998 la date d'entrée en vigueur de la sLoi sur le pare marin du Saguenay-Saint-Laurent, C.P. 1998-963 (1998), 132s, Gaz Can., Ptie II, n ${ }^{\circ} 13$, p. 1941. Décret concernant l'entrée en vigueur de la * Loi sur le pare marin du SaguenaySaint-Laurent, Décret 782-98, 10 juin 1998. $130 \%, G, O, Q$, Ptie 2, n² 26, p. 3183.

6 Le parc s"étend jusqu' a la ligne des hautes marées, sans inondation ni débordement. Sont exclues les propriêtés non détenues par lé gouvernement du Québec, les infrastructures maritimes, les îles et îlots (Ministère du Patrimoine canadien, ministère de l' Environnement et de la faune du Québec, 1995 : 11).

7 Loi fédérale sur le parc marin du SaguenaySaint-Laurent, art. 4.

8 Les recherches globales sur la nature physicochimique, le mouvement des eaux, la population animale et végétale n'ont étế entreprises qu'en 1991 (Hamel, 1996: 16).

9 Cinquante bateaux de croisière détiennent des permis de navigation (Vézina, $1997 ; 27$ ).

\section{BIBLIOGRAPHIE}

Agardy, M.T. (1993), a Accomodating Ecotourism in Multiple Use Planning of Coastal and Marine Protected Areas *, Ocean and Coastal Management, 20, 3, p. 19.

Alexander, L.M. (1993), \& Large Marine Ecosystems. A New Focus for Marine Resources Management $₫$, Marine Policy, 17, 3, p. 186.

Boo, E. (1991), \& Planning for Ecotourism s;, Parks, 2, 3, p. 4.

Breton, M. et coll. (1990), Le beluga, Collection Le monde sous-marin, Ministère des Pêches et océans.

Butler, M. (1994), * When You Can't Build Fences; The West Isles Marine Park Proposal $»$, dans Lamson, C. (Ed.), The Sea Has Many Voices, McGill-Queen's University Press, p. 125-134.

Centre Saint-Laurent (1991), Le parc marin du Saguenay... Un milieu unique au sein d"wn continent, Bilan Saint-Laurent, juin.

Environnement Canada (1987), Politique sur les parcs marins nationaux.

Fiske, S.J. (1992), "Sociocultural Aspects of Establishing Marine Protected Areas *, Ocean and Coastal Management, 18, p. 25.

Hamel, A. (1996), La survie du béluga et le zonage da parc marin du Saguenay, mémoire de maîtrise en Sciences de l'environnement, UQAM.

Inskeep. E. (1991), Tourism Planning; an Integrated and Sustainable Development Approach, p. 418-420.

Leitmann, J. (1998), \& Options for Managing Protected Areas: Lessons from International Experience », Journal of Environmental Planning and Management, 41, 1, p. 129-143.

Maltais, B. (1995), \& Le parc marin du Saguenay-Saint-Laurent », Téoross, 14, 1, p. 54.

Miller, M. L. (1993), * The Rise of Coastal and Marine Tourism $;$ Ocean and Coastal Management, 20, p, 181-189.

Ministère du Patrimoine canadien (1994), Principes directeurs et politiques de gestion.

Ministère du Patrimoine canadien (1995), D'un océan à l'autre, Plan de réseau des aires marines de conservation du Canada, $108 \mathrm{p}$.

Ministère du Patrimoine canadien (1997), Plan de réseau des pares nationaux.

Ministère du Patrimoine canadien, ministère de l'Environnement et de la Faune du Québec (1995). Le parc marin du Saguenay-Saint-Laureni, Le plan directeur.

O.C.D.E. (1989), Politique du tourisme et tourisme international.

Vézina, A. (1997), * L'écotourisme: pile et face *, Franc-Vert, juin-juillet, p. 26. 